\title{
A Simplified Control Approach for the Neuromuscular Blockade Level
}

\author{
Juliana Almeida ${ }^{1,3}$, Teresa Mendonça ${ }^{2,3}$, and Paula Rocha ${ }^{1,3}$ \\ 1 Universidade do Porto, Faculdade de Engenharia, \\ Rua Dr. Roberto Frias, s/n 4200-465 Porto, Portugal \\ almeidajfc@gmail.com, mprocha@fe.up.pt \\ 2 Universidade do Porto, Faculdade de Ciências, \\ Rua do Campo Alegre, s/n 4169-007 Porto, Portugal \\ tmendo@fc.up.pt \\ 3 Research Center for Systems and Technologies (SYSTEC), \\ Rua Dr. Roberto Frias, s/n 4200-465 Porto, Portugal
}

\begin{abstract}
In this paper a new simplified control scheme for the neuromuscular blockade level that only requires the knowledge of one model parameter is proposed. The control law is designed to track a desired target neuromuscular blockade level. Furthermore, an identification procedure to obtain the necessary model parameter is implemented. The results were validated by simulations based on real data collected during surgeries.
\end{abstract}

Keywords: Positive control law, Neuromuscular level, General anesthesia

\section{Introduction}

The impact of automatic control and system identification techniques on devices and applications in the field of biomedicine has recently been growing. One example of application of control to biomedical systems is the automatic control of anesthetics during general surgery. In this case, several signals are taken into account to monitor the patient. One of these signals is the neuromuscular blockade (NMB) level, which gives information about the patient's state regarding muscle paralysis, induced to allow the intubation and other clinical procedures. The degree of the neuromuscular blockade can be assessed by applying a train-of-four of supramaximal twitch stimulus to the hand peripheral nerve. The NMB level corresponds to the first response measured and varies between 100\% (normal muscle activity) and $0 \%$ (full paralysis). To achieve an adequate NMB level muscle relaxants are administered, e.g. rocuronium or atracurium. Mathematically, the relation between the drug dose and its effect can be modelled by a pharmacokinetic/pharmacodynamic (PK/PD) model. The pharmacokinetic model describes the time course of the drug concentration in the plasma whereas the pharmacodynamic model studies the relation between the drug concentration and its 
effect. This latter relates the effect concentration with the response by a nonlinear equation known as Hill equation, [1]. However, PK/PD models have the drawback of needing a large number of parameters to characterize the patient. In order to overcome this drawback a new simplified SISO Wiener model proposed in [2] is used in this paper. This model involves a number minimal of patientdependent parameters. More concretely, it has one parameter in the linear part of model and another one in the nonlinear part. Here, a new simplified control law that only requires knowledge of the parameter associated to the nonlinear model is proposed. This control law is based on the one proposed in [3], which has already been applied to control the NMB level, using the two model parameters [4], [5]. In order to implement our law, an estimation procedure for the only parameter on which it depends is also proposed.

This paper is organized as follows. In Section 2 the SISO Wiener model and the new control law are described. The main results are shown in Section 3, and the conclusions are drawn in Section 4.

\section{One-parameter Control law}

This section presents our new positive control law for the NMB level that uses a simplified description of the system. The underlying SISO Wiener NMB model is first introduced.

\subsection{SISO Wiener model}

The NMB model proposed in [2] for the description of the effect of the muscle relaxant in the human body consists of one linear model followed by a nonlinear static equation.

The linear dynamics is modeled by

$$
C_{e}(s)=\frac{k_{1} k_{2} k_{3} \alpha^{3}}{\left(s+k_{1} \alpha\right)\left(s+k_{2} \alpha\right)\left(s+k_{3} \alpha\right)} U(s),
$$

where $C_{e}(s)$ is the Laplace transform of the effect concentration $c_{e}(t) ; U(s)$ is the Laplace transform of the input drug dose $u(t) ; k_{1}, k_{2}$ and $k_{3}$ are parameters that have been suitably determined in [2] for the muscle relaxants rocuronium and atracurium, and $\alpha>0$ is patient-dependent parameter.

The state-space representation of the model is

$$
\left\{\begin{aligned}
\dot{x}(t) & =\alpha A x(t)+\alpha B u(t) \\
c_{e}(t) & =\left[\begin{array}{lll}
0 & 0 & 1
\end{array}\right] x(t)
\end{aligned}\right.
$$

where the matrix $A$ and the vector $B$ are defined as 


$$
A=\left[\begin{array}{ccc}
-k_{3} & 0 & 0 \\
k_{2} & -k_{2} & 0 \\
0 & k_{1} & -k_{1}
\end{array}\right], \quad B=\left[\begin{array}{c}
-k_{3} \\
0 \\
0
\end{array}\right]
$$

The nonlinear static equation to describe the relation between the effect concentration and the actual drug effect is given by the following Hill's equation

$$
y(t)=\frac{100}{1+\left(\frac{c_{e}(t)}{C_{50}}\right)^{\gamma}}
$$

where $C_{50}$ is the half of the maximum effect concentration and has fixed value according $[6] ; \gamma$ is the only other patient-dependent parameter.

\subsection{Control law for the NMB level}

The control law used in this paper is basically the one introduced in [3], which applied to control the NMB level in [4] and [5]. The difference is that, here, the controller is independent from the parameter $\alpha$, which constitutes a simplification with respect to the previous approaches. Both the original and our simplified law have the convergence of the total system mass $M(x)=\sum_{i=1}^{3} x_{i}$ to a desired system mass $M^{*}$ as control objective. So, our aim of tracking a desired NMB level is achieved by reaching and maintaining an appropriate mass of drug in the patient's body (or system). More concretely, the simplified control law obtained is

$$
\left\{\begin{array}{l}
u(t)=\max (0, \tilde{u}(t)) \\
\left.\left.\tilde{u}(t)=-\left(\sum_{i=1}^{3} b_{i}\right)^{-1}\left[\begin{array}{lll}
1 & 1 & 1
\end{array}\right]\right) A x(t)+\lambda\left(M(x)-M^{*}\right)\right]
\end{array}\right.
$$

with $\lambda>0$ being a design parameter. Note the absence of the parameter $\alpha$ from (2). The parameter $\gamma$ from (3) is implicitely present as it is used to compute $M^{*}$.

The proof that this control law achieves its mass tracking goal is similar to the one given in [3]. Here, just to give an idea of how it works, we consider the simple case when $\tilde{u}(t) \geq 0$. In this case, letting $\Delta M=M(x)-M^{*}$ : 


$$
\begin{aligned}
\overbrace{\Delta M}^{\dot{v}} & =\dot{M}(x)=\left[\begin{array}{lll}
1 & 1 & 1
\end{array}\right] \dot{x}(t) \\
& =\alpha\left[\begin{array}{lll}
1 & 1 & 1
\end{array}\right] A x(t)+\alpha\left(\sum_{i=1}^{3} b_{i}\right) \times \\
& \left.\times\left(-\left(\sum_{i=1}^{3} b_{i}\right)^{-1}\left(\begin{array}{lll}
1 & 1 & 1
\end{array}\right] A x(t)+\lambda\left(M(x)-M^{*}\right)\right)\right) \\
& =-\alpha \lambda\left(M(x)-M^{*}\right) \\
& =-\alpha \lambda \Delta M=-\lambda_{\alpha} \Delta M
\end{aligned}
$$

Since $\alpha$ and $\lambda$ are positive, $-\lambda_{\alpha}<0$ and hence $\Delta M$ converges to zero, i.e., $M(x)$ converges to $M^{*}$.

Thus, the patient parameter $\alpha$ may indeed be left out of the control law for the rocuronium mass. Although $\alpha$ influences the in the speed of convergence, this can be compensated by the design parameter $\lambda$. As can be seen in (5) the controller action just depends on a suitable choice of the value for the desired mass $M^{*}$. To compute this value, the equilibrium point of the closed-loop system, when $M(x)=M^{*}$ was determined and the steady-state effect concentration $c_{e}$ was shown to be equal to $\frac{M^{*}}{3}$. This enables to determine the value of the desired mass $M^{*}$ by the inversion of the Hill equation (3) for a desired set-point value $y^{*}$ for the NMB level. According the clinicians, during general anesthesia, this desired value should be equal to $10 \%$.

Indeed, we have

$$
M^{*}=3 \times\left(\frac{100}{y^{*}}-1\right)^{1 / \gamma} C_{50}=3 \times 9^{1 / \gamma} C_{50}
$$

Thus, once the patient-dependent parameter $\gamma$ is known, the value of $M^{*}$ is obtained by equation (6).

\subsection{Parameter estimation}

Although, the controller action does not depend from the parameter of the linear model, the determination of the suitable mass $M^{*}$ depends on the parameter $\gamma$. In order to obtain the value of this parameter, a simple procedure for parameter estimation will be performed. Note that, $\gamma$ appears in the Hill equation,

$$
y(t)=\frac{100}{1+\left(\frac{c_{e}(t)}{C_{50}}\right)^{\gamma}}
$$

This parameter is identified when the controller action begins which corresponds to time instant of the initial recovery after an initial bolus. This time of 
recovery, $t^{*}$, is given by the algorithm OLARD [8]. So, at this time instant, an estimate $\hat{\gamma}$ of $\gamma$ is obtained by the inversion of the Hill equation

$$
\hat{\gamma}=\frac{\log \left(\frac{100}{y\left(t^{*}\right)}-1\right)}{\log \left(\frac{c_{e}\left(t^{*}\right)}{C_{50}}\right)}
$$

\section{Simulation results}

This section presents the results obtained by the application of the control scheme proposed here to control the NMB level via the administration of the muscle relaxant rocuronium. Fifty real cases were collected using the Galeno platform developed in the framework of the Portuguese funding agency (FCT) project Galeno, that incorporates several identification and control procedures for automation in the administration of anesthetics. Furthermore, a Prediction Error Method was used to identify the patient-dependent parameters: $\theta_{i}=$ $\left(\alpha_{i}, \gamma_{i}\right), i=1, \ldots, 50$. Here, the values for the parameters corresponding to the patient number seven were used to represent the real patient and to obtain the real response: $\theta_{7}=(0.0293,1.4728)$. The values of $k_{1}, k_{2}$ and $k_{3}$ are fixed
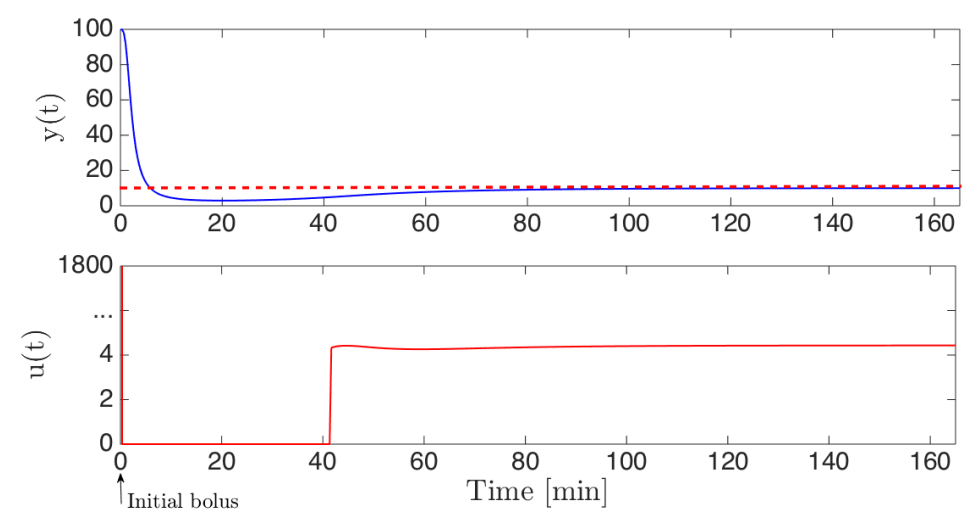

Fig. 1. NMB level evolution obtained with the application of the proposed control scheme applied at $t^{*}=41.3 \mathrm{~min}$.

and equal to 1, 9 and 10 respectively, and the values of $C_{50}$ is 1 [7]. The value for the design parameter $\lambda_{\alpha}$ was taken as 0.2 .

Since for simplicity, we chose not to identify the parameter $\alpha$, the control scheme is designed taking into account its mean value $\alpha: \bar{\alpha}=0.0307$.

Moreover the identification of the parameter $\gamma$ of the nonlinear model at the time instant $t^{*}=41,3 \mathrm{~min}$ is performed as mentioned in equation (8). The value of the estimated parameter is $\hat{\gamma}=1.4755$. The proposed control scheme is applied from the time instant $t^{*}$ on considering the obtained estimated for the 
parameter $\gamma$. Fig. 1 shows the patient response and the input signal obtained by applying the described strategy.

To analyze the performance of the identification procedure, the controlled NMB response was obtained considering the following scenarios:

1. $\bar{\alpha}, \bar{\gamma}$, i.e., mean values for $\alpha$ and $\gamma(\bar{\gamma}=1.8499)$;

2. $\bar{\alpha}, \hat{\gamma}$, i.e., mean value for $\alpha$ and the estimate obtained by the identification procedure for $\gamma$.

The corresponding results can be observed in Fig. 2. As it is possible to see,

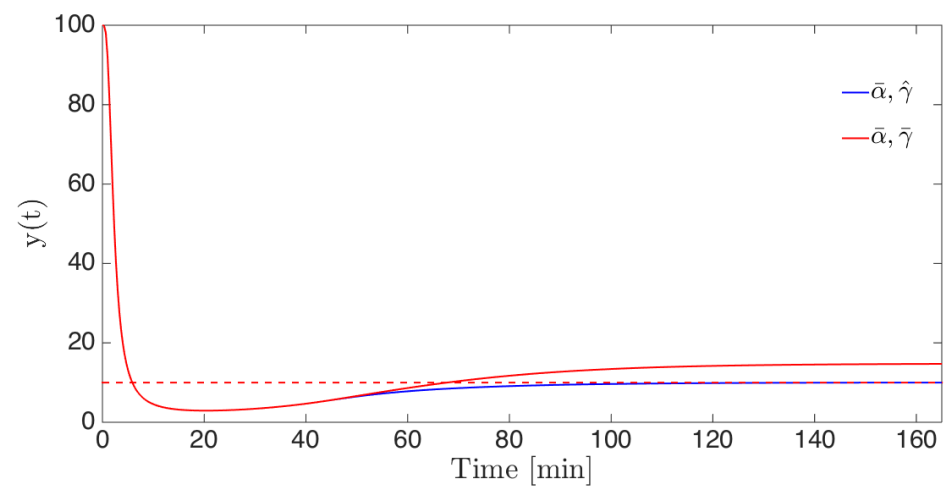

Fig. 2. NMB level evolution for the scenarios (1) and (2) when the control law is applied at $t^{*}=41.3 \mathrm{~min}$.

with our identification procedure the NMB level tracks the desired NMB level of $10 \%$.

In order to analyze the performance of the control scheme under a more realistic scenario, a noise signal was added to the real response. The noise signal was obtained from a typical NMB real case. For this scenario, the value of $\hat{\gamma}$ is 1.3660. Fig. 3 shows the NMB level evolution and the input signal when noise is considered.

\section{Conclusions}

In this paper a new simplified control scheme for the neuromuscular blockade that only requires the knowledge of the parameter of the nonlinear part of the model is proposed. The control law is designed to control the amount of the muscle relaxant that should be administered during a general anesthesia to track a desired NMB level of $10 \%$. In order to obtain the aforementioned parameter, an identification procedure based on the patient's response was also proposed. The results were validated by simulations based on real collected data; although this is a preliminary study, the result are encouraging. 


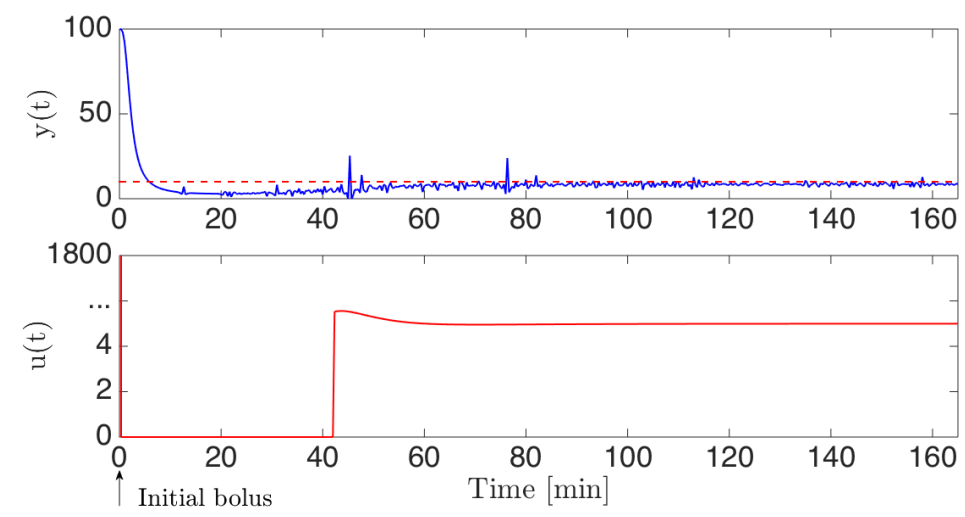

Fig. 3. NMB level evolution obtained with the application of the proposed control scheme at $t^{*}=41.3 \mathrm{~min}$ when noise is added to the real signal.

\section{Acknowledge}

The author Juliana Almeida acknowledge the support from FCT Fundação para a Ciência e Tecnologia under the SFRH/BD/87128/2012.

\section{References}

1. W. Haddad, V. Chellaboina, Q. Hui: Nonnegative and Compartmental Dynamical Systems Princeton University Press, 2010

2. M. M. da Silva, T. Wigren, T. Mendonça: Nonlinear Identification of a Minimal Neuromuscular Blockade Model in Anesthesia IEEE Trans. Contr. Sys. Techn., 20, pp. 181-188, 2012

3. G. Bastin, A. Provost: Feedback stabilisation with positive control of dissipative compartmental systems Proceeding of the 15th Internation Symposium on Mathematical Theory of Networks ans Systems, 2002

4. J. Almeida, M. M. da Silva, T. Mendoną, P. Rocha: A compartmental model-based control strategy for NeuroMuscular Blockade level Proc. 18th IFAC World Congress, pp. 599-604, 2011

5. J. Almeida, T. Mendonça, P. Rocha: An improved strategy for NeuroMuscular Blockade control with parameter uncertainty 50th IEEE Conference on Decision and Control and European Control Conference (CDC-ECC), pp. 867 - 872, 2011

6. H. Alonso, T. Mendona, P. Rocha: A hybrid method for parameter estimation and its application to biomedical systems Computer Methods and Programs in Biomedicine 89 (2) 112 - 122, 2008

7. M.M. Silva, J.M. Lemos, A. Coito, B.A. Costa, T. Wigren, T. Mendonça: Local identifiability and sensitivity analysis of neuromuscular blockade and depth of hypnosis models Computer Methods and Programs in Biomedicine, 113 (1), 23 - 36, 2014

8. M.M. Silva, C. Sousa, R. Sebastiao, J. Gama, T. Mendonca, P. Rochak, S. Esteves: Total Mass TCI driven by Parametric Estimation Proc. 17th Mediterranean Conference on Control \& Automation, 1149-1154, 2009 